\title{
Neimark-Sacker bifurcation and the generate cases of Kopel oligopoly model with different adjustment speed
}

\author{
Bo Li ${ }^{1,2^{*}}$ (D), Qizhi He$e^{2}$ and Ruoyu Chen ${ }^{1,2}$
}

\author{
"Correspondence: libo@aufe.edu.cn; \\ libomaths@163.com \\ 'Business School, Nanjing \\ University, Nanjing, People's \\ Republic of China \\ ${ }^{2}$ School of Finance, Anhui University \\ of Finance and Economics, Bengbu, \\ People's Republic of China
}

\begin{abstract}
In this paper, bifurcations and chaotic behaviours of Kopel oligopoly model with different adjustment speed are discussed. The results imply that the Kopel oligopoly model undergoes flip bifurcation, Neimark-Sacker bifurcation, 1:3 and 1:4 resonances, which could induce complex dynamics, especially global behaviours between different orbits. The conditions for the occurrence of three different kinds of bifurcation are derived. Furthermore, the numerical simulations provide us the case study of theoretical analysis and the corresponding dynamical behaviours, especially the occurrence of global orbits.
\end{abstract}

Keywords: Kopel model; Adjustment speed; Neimark-Sacker bifurcation; 1:3 and 1:4 resonances; Homoclinic orbits

\section{Introduction}

In theory, there exist three types of market structure: perfect competition, oligopoly and monopoly; oligopoly is popular in practice, especially duopoly. As the earliest economist of oligopoly theory, Cournot [8] constructed the famous oligopoly model based on some idealised assumptions and described the influences on each other from the viewpoint of competition in production. The early oligopoly is criticised for the over idealised assumptions, but oligopoly theory has already been perfected gradually. Taking the agents' learning mechanism into account, Kopel [17] proposed a revised Cournot model (known as Kopel oligopoly model in this paper) for more reasonable interpretation of actual competition between two oligopolists. The Kopel oligopoly model can be described as that in the following map:

$$
\left(\begin{array}{l}
x \\
y
\end{array}\right) \mapsto\left(\begin{array}{l}
\left(1-\rho_{1}\right) x+\rho_{1} \mu_{1} y(1-y) \\
\left(1-\rho_{2}\right) y+\rho_{2} \mu_{2} x(1-x)
\end{array}\right)
$$

where $x$ and $y$ are the quantities of two oligopolists $\mathrm{X}$ and $\mathrm{Y}$, respectively. $\rho_{1(2)}$ is called the adjustment coefficients to allocate the weight between the previous production and the optimal production according to the logistic reaction functions. $\mu_{1(2)}$ is chosen to measure

(c) The Author(s) 2020. This article is licensed under a Creative Commons Attribution 4.0 International License, which permits use, sharing, adaptation, distribution and reproduction in any medium or format, as long as you give appropriate credit to the original author(s) and the source, provide a link to the Creative Commons licence, and indicate if changes were made. The images or other third party material in this article are included in the article's Creative Commons licence, unless indicated otherwise in a credit line to the material. If material is not included in the article's Creative Commons licence and your intended use is not permitted by statutory regulation or exceeds the permitted use, you will need to obtain permission directly from the copyright holder. To view a copy of this licence, visit http://creativecommons.org/licenses/by/4.0/. 
the intensity of the effect that the rival imposed on itself. Moreover, the existence and stability of "Nash-equilibria" are proved and invariant curve and strange attractor are plotted to show the complexity of competition between the rivals. Many economists and applied mathematicians studied the interests and dynamics of the Kopel oligopoly model with different methods. More attention was paid to bifurcation and chaos of map (1) by Agiza [1], and OGY method was introduced to control the chaos for improving the market's performance. With the help of MATCONTM, a set of package on the basis of MATLAB developing platform, Govaerts and Khoshsiar Ghaziani [12] used the method of bifurcation continuation to study the one (two)-parameter bifurcations. A revised Kopel oligopoly model with extrapolative foresight was constructed by Gao, Zhong and Mei [11], and Neimark-Sacker bifurcation analysis showed the complex dynamics and the transitions between different dynamical systems. See more research about Kopel oligopoly model in $[3,5,10,30,31,35]$.

As presented above in the related research, dynamical system theory was introduced as an important tool to explore the dynamics in oligopoly theory. Colomobo and Labrecciosa [6] considered different competition cases between multiple oligopolists and different feedbacks, which corresponds to different types of equilibria (Cournot equilibrium and Stackelberg equilibrium); they were proved to be efficient differently, especially in the short-run terms and at the steady-state. Matouk, Elsadany and Xin [24] established a quadropoly Cournot game model with heterogeneous players and complex dynamics, especially chaos; they were analysed through supercritical Neimark-Sacker bifurcation and flip bifurcation at the fixed point. In [14], the authors introduced a kind of evolutionary competition of Cournot oligopoly game and discussed the relations between the instability threshold and the number of firms involved. Complex dynamics of Cournot model with asymmetric information were investigated by $\mathrm{Yu}$ and $\mathrm{Yu}$ [36]. Bifurcation and chaos were studied with respect to probability parameter $\theta$. Furthermore, chaos control was carried out to improve the performance for specific market. Ma, Yang and Liu [23] introduced the carbon emission reduction constraint and analysed the influence on oligopolists. The results show us that the new environmental regulation is a chance for expressing its welfare implications and changing competitive status. For more systematic introduction and recent advances, one can refer to [2, 4-7, 9, 18, 25-28, 32, 39]. In fact, bifurcation theory, as one of the most powerful analytical tools, can be applied in many applied sciences, such as economic models, predator-prey models, neural networks. One can find more useful information on bifurcation analysis and the applications in [15, 16, 20-22, 29, 37, 38].

In this paper, we suppose that the influences between each other are the same and get the following map:

$$
\left(\begin{array}{l}
x \\
y
\end{array}\right) \mapsto\left(\begin{array}{l}
\left(1-\rho_{1}\right) x+\rho_{1} \mu y(1-y) \\
\left(1-\rho_{2}\right) y+\rho_{2} \mu x(1-x)
\end{array}\right) .
$$

When $\rho_{1}=\rho_{2}$, some research was carried out and the dynamics derived showed the complex and interesting economic interests. Here, we consider the more general case and the corresponding dynamics. In this paper, the aim is to show that map (2) possesses Neimark-Sacker bifurcation, 1:3 and 1:4 resonances, from which different types of dynamics for two agents are derived and that means the complex competition between them, such as the occurrence of homoclinic behaviours. When discussing the Neimark-Sacker 
bifurcation, $\mu$ is chosen to illustrate the changes of output between each other. As for 1:3 and 1:4 resonances, another parameter $\rho_{1}$ is introduced to analyse the dynamics, especially describing the transitions between different types of dynamical behaviours for two agents. One can show that the analysis process is similar as that for $\rho_{1}$ when parameter $\rho_{2}$ is introduced.

This paper is outlined as follows. The existence and local dynamics of fixed points for map (2) are investigated as the general case of results in [12], especially the conditions of Neimark-Sacker bifurcation, 1:3 and 1:4 resonances in Sect. 2. In Sects. 3, 4 and 5, we presented the deductions and discuss the conditions of occurrence and the corresponding dynamics for Neimark-Sacker bifurcation, 1:3 and 1:4 resonances, respectively. Three case studies are provided for the bifurcation analysis and some new and interesting phenomena are observed in Sect. 5. Finally, a brief discussion including economic interest and further research concludes the paper in Sect. 6.

\section{Existence and stability of fixed points of map (2)}

To get the explicit expressions of fixed points for map (2), we need to solve the following equations:

$$
\left\{\begin{array}{l}
x=\mu y(1-y), \\
y=\mu x(1-x) .
\end{array}\right.
$$

After complex computations, we get the following results about fixed points of map (2) as those in [17].

\section{Lemma 2.1}

(i) If $\mu>0$, there always exists a trivial fixed point $E_{0}(0,0)$ for map (2);

(ii) If $\mu \geq 1$, there always exists a positive fixed point $E_{1}\left(x_{1}, y_{1}\right)$ for map (2), where $x_{1}=y_{1}=1-\frac{1}{\mu}$

(iii) If $1<\mu \leq 3$, there exists a fixed point $E_{2}\left(x_{2}, y_{2}\right)$ and its symmetric point $E_{3}\left(y_{2}, x_{2}\right)$ for map (2), where $x_{2}=\frac{\mu+1+\sqrt{\mu^{2}-2 \mu-3}}{2 \mu}$ and $y_{2}=\frac{\mu+1-\sqrt{\mu^{2}-2 \mu-3}}{2 \mu}$.

See Fig. 1 for numerical solutions. The solutions in red, blue, green and yellow colours are $E_{0}, E_{1}, E_{2}$ and $E_{3}$ presented in the above lemma, respectively.

Figure 1 The existing solution with respect to $\mu$

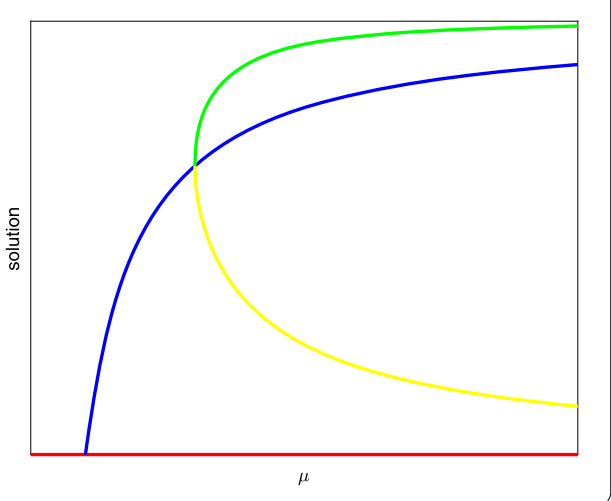


The trivial fixed point $E_{0}$ is of no interest in economics and the dynamics of $E_{3}$ is similar to $E_{2}$. In this research, the dynamics of $E_{1}$ and $E_{2}$ are explored in detail. To investigate the stability of $E_{1}$ and $E_{2}$, the following Jacobian matrix is derived respectively:

$$
J(x, y)=\left(\begin{array}{cc}
1-\rho_{1} & \rho_{1} \mu(1-2 y) \\
\rho_{2} \mu(1-2 x) & 1-\rho_{2}
\end{array}\right)
$$

and the corresponding characteristic equation is

$$
F(E)=F(x, y)=\lambda^{2}-\left(2-\rho_{1}-\rho_{2}\right) \lambda+\left(1-\rho_{1}\right)\left(1-\rho_{2}\right)-\mu^{2} \rho_{1} \rho_{2}(1-2 x)(1-2 y)=0
$$

at $E(x, y)$.

Lemma 2.2 Define $\mu_{0}=\sqrt{1+\frac{4-2 \rho_{1}-2 \rho_{2}}{\rho_{1} \rho_{2}}}$ and for $E_{0}$ of map (2):

(i) if $0<\mu<1$ and $2\left(\rho_{1}+\rho_{2}\right)-4<\rho_{1} \rho_{2}\left(1-\mu^{2}\right)<\rho_{1}+\rho_{2}$, it is a sink;

(ii) if $\mu=1$, it is nonhyperbolic and a fold bifurcation occurs at $E_{0}$;

(iii) if $\rho_{1}+\rho_{2}<2$ and $1<\mu<\mu_{0}$, it is a saddle;

(iv) if $\rho_{1}+\rho_{2}<2$ and $\mu=\mu_{0}$, it is nonhyperbolic and a flip bifurcation occurs at $E_{0}$.

Lemma 2.3 Define $\mu_{11}=2+\sqrt{1+\frac{4-2 \rho_{1}-2 \rho_{2}}{\rho_{1} \rho_{2}}}, \mu_{12}=2-\sqrt{1+\frac{4-2 \rho_{1}-2 \rho_{2}}{\rho_{1} \rho_{2}}}$ and for $E_{1}$ of map (2):

(i) if $1<\mu<3$ and $2\left(\rho_{1}+\rho_{2}\right)-4<\rho_{1} \rho_{2}\left(4 \mu-\mu^{2}-3\right)<\rho_{1}+\rho_{2}$, it is a sink;

(ii) if $1<\mu<3$ and $\rho_{1} \rho_{2}\left(4 \mu-\mu^{2}-3\right)<2\left(\rho_{1}+\rho_{2}\right)-4$, it is a saddle;

(iii) if $1<\mu<3$ and $\max \left\{2\left(\rho_{1}+\rho_{2}\right)-4, \rho_{1}+\rho_{2}\right\}<\rho_{1} \rho_{2}\left(4 \mu-\mu^{2}-3\right)$, it is a source;

(iv) if $\mu=3$, it is nonhyperbolic and a fold bifurcation occurs at $E_{1}$;

(v) if $\mu=\mu_{11}\left(\mu_{12}\right)$, it is nonhyperbolic and a flip bifurcation occurs at $E_{1}$.

When $\mu$ becomes bigger, different types of stability for $E_{0}\left(E_{1}\right)$ occur. For the case of fold bifurcation, some facts can be found in Lemma 2.1, that is, there exist two newborn fixed points when $\mu$ varies in the neighbourhood of 1 or 3 , respectively. For the case of flip bifurcation, theoretical analysis and numerical simulation can be carried out as those done in [12].

Lemma 2.4 Define $\mu_{21}=1+\sqrt{4+\frac{2\left(\rho_{1}+\rho_{2}-2\right)}{\rho_{1} \rho_{2}}}, \mu_{22}=1+\sqrt{4+\frac{1}{\rho_{1}}+\frac{1}{\rho_{2}}}$ and for $E_{2}$ of map (2):

(i) if $\mu=3$, it is nonhyperbolic and a fold bifurcation occurs at $E_{2}$;

(ii) if $\max \left\{3, \mu_{21}\right\}<\mu<\mu_{22}$, it is a sink;

(iii) if $3<\mu<\mu_{21}$, it is a saddle;

(iv) if $\mu>\max \left\{3, \mu_{21}, \mu_{22}\right\}$, it is a source;

(v) if $\mu=\mu_{21}$, it is nonhyperbolic and a flip bifurcation occurs at $E_{2}$;

(vi) if $\mu=\mu_{22}$, it is nonhyperbolic and a Neimark-Sacker bifurcation occurs at $E_{2}$. Moreover,

(vi1) if $\mu=\mu_{22}$ and $\rho_{1}=\rho_{10}:=3-\rho_{2}, a 1: 3$ resonance occurs at $E_{2}$;

(vi2) if $\mu=\mu_{22}$ and $\rho_{1}=\rho_{11}:=2-\rho_{2}, a 1: 4$ resonance occurs at $E_{2}$.

The occurrence of fold (flip) bifurcation of Lemma 2.4 is similar as the corresponding cases of Lemma 2.3. Neimark-Sacker bifurcation is extremely interesting in economics, 
because that means the stable periodic behaviours for agents. We will pay more attention to Neimark-Sacker bifurcation and two important degenerate types, 1:3 and 1:4 resonances.

\section{Neimark-Sacker bifurcation for $E_{2}$}

Based on case (vi) in Lemma 2.4, $\mu$ is chosen as a bifurcation parameter to analyse Neimark-Sacker bifurcation and related dynamics at $E_{2}$.

In what follows, we investigate the Neimark-Sacker bifurcation of $E_{2}$ if the parameter $\mu$ varies in the neighbourhood of $\mu_{22}$. We consider map (2) as follows:

$$
\left(\begin{array}{l}
x \\
y
\end{array}\right) \mapsto\left(\begin{array}{l}
\left(1-\rho_{1}\right) x+\rho_{1} \mu_{22} y(1-y) \\
\left(1-\rho_{2}\right) y+\rho_{2} \mu_{22} x(1-x)
\end{array}\right) .
$$

Choosing $\bar{\mu}$ as a parameter for bifurcation analysis, we consider the following perturbation map:

$$
\left(\begin{array}{l}
x \\
y
\end{array}\right) \mapsto\left(\begin{array}{c}
\left(1-\rho_{1}\right) x+\rho_{1}\left(\mu_{22}+\bar{\mu}\right) y(1-y) \\
\left(1-\rho_{2}\right) y+\rho_{2}\left(\mu_{22}+\bar{\mu}\right) x(1-x)
\end{array}\right),
$$

where $|\bar{\mu}| \ll 1$.

Let $\breve{x}=x-x_{2}$ and $\breve{y}=y-y_{2},\left(x_{2}, y_{2}\right)$ be transformed to the origin. Then we get the following map, which is topological equivalent to map (6):

$$
\left(\begin{array}{c}
\breve{x} \\
\breve{y}
\end{array}\right) \mapsto\left(\begin{array}{c}
\left(1-\rho_{1}\right) \breve{x}+a_{20}(\bar{\mu}) \breve{y}+\rho_{1} y_{2}\left(1-y_{2}\right) \bar{\mu}-\rho_{1}\left(\mu_{22}+\bar{\mu}\right) \breve{y}^{2} \\
\left(1-\rho_{2}\right) \breve{y}+a_{21}(\bar{\mu}) \breve{x}+\rho_{2} x_{2}\left(1-x_{2}\right) \bar{\mu}-\rho_{2}\left(\mu_{22}+\bar{\mu}\right) \breve{x}^{2}
\end{array}\right)
$$

where

$$
\begin{aligned}
& a_{20}(\bar{\mu})=\rho_{1}\left(1-2 y_{2}\right)\left(\mu_{22}+\bar{\mu}\right), \\
& a_{21}(\bar{\mu})=\rho_{2}\left(1-2 x_{2}\right)\left(\mu_{22}+\bar{\mu}\right) .
\end{aligned}
$$

The corresponding characteristic polynomial of Jacobian matrix for map (7) at the origin is

$$
\lambda^{2}+p(\bar{\mu}) \lambda+q(\bar{\mu})
$$

where

$$
\begin{aligned}
& p(\bar{\mu})=-2+\rho_{1}+\rho_{2}, \\
& q(\bar{\mu})=\left(1-\rho_{1}\right)\left(1-\rho_{2}\right)-\rho_{1} \rho_{2}\left(\mu_{22}+\bar{\mu}\right)^{2}\left(1-2 x_{2}\right)\left(1-2 y_{2}\right) .
\end{aligned}
$$

It is clear that the corresponding eigenvalues at the origin are $\lambda$ and its conjugate $\bar{\lambda}$, where

$$
\lambda, \bar{\lambda}=1-\frac{\rho_{1}+\rho_{2}}{2} \pm \frac{i}{2} \sqrt{4 q(\bar{\mu})-p^{2}(\bar{\mu})},
$$


and there exist

$$
|\lambda|=\sqrt{q\left(\mu_{2}\right)}=1, \quad l=\left.\frac{d|\lambda|}{d \bar{\mu}}\right|_{\bar{\mu}=0}=-\frac{\rho_{1}+\rho_{2}}{2}<0 .
$$

Clearly, $p(\bar{\mu})=-2+\rho_{1}+\rho_{2}<0 \neq 0,1$, then we have $\lambda^{n}\left(\mu_{2}\right) \neq 1, n=1,2,3,4$.

Let

$$
\left(\begin{array}{l}
\breve{x} \\
\breve{y}
\end{array}\right)=\left(\begin{array}{cc}
a_{20} & 0 \\
\frac{3 \rho_{1}+\rho_{2}}{2} & \frac{\sqrt{\left(\rho_{1}+\rho_{2}\right)\left(4-\rho_{1}-\rho_{2}\right)}}{2}
\end{array}\right)\left(\begin{array}{l}
u \\
v
\end{array}\right),
$$

then map (7) becomes

$$
\left(\begin{array}{l}
u \\
v
\end{array}\right) \mapsto\left(\begin{array}{cc}
\operatorname{Re}(\lambda) & \operatorname{Im}(\lambda) \\
-\operatorname{Im}(\lambda) & \operatorname{Re}(\lambda)
\end{array}\right)\left(\begin{array}{l}
u \\
v
\end{array}\right)+\left(\begin{array}{l}
f(u, v) \\
g(u, v)
\end{array}\right)
$$

where

$$
\begin{aligned}
& \operatorname{Re}(\lambda)=1-\frac{\rho_{1}+\rho_{2}}{2}, \\
& \operatorname{Im}(\lambda)=\frac{\sqrt{\left(\rho_{1}+\rho_{2}\right)\left(4-\rho_{1}-\rho_{2}\right)}}{2}, \\
& f(u, v)=\left[\left(3 \rho_{1}+\rho_{2}\right) u+2 \operatorname{Im}(\lambda) v\right]^{2} h_{n s}, \\
& g(u, v)=-\frac{\left(3 \rho_{1}+\rho_{2}\right)}{2 \operatorname{Im}(\lambda)}\left[\left(3 \rho_{1}+\rho_{2}\right) u+2 \operatorname{Im}(\lambda) v\right]^{2} h_{n s}-\frac{\rho_{2} a_{20}^{2}}{\operatorname{Im}(\lambda)} u^{2}, \\
& h_{n s}=-\frac{\mu_{22} \rho_{1}}{4 a_{20}},
\end{aligned}
$$

and

$$
\begin{aligned}
& f_{u u}=\left(3 \rho_{1}+\rho_{2}\right)^{2} h_{n s}, \quad f_{v v}=4 h \operatorname{Im}^{2}(\lambda), \quad f_{u v}=4 h\left(3 \rho_{1}+\rho_{2}\right) \operatorname{Im}(\lambda), \\
& g_{u u}=-\frac{\left(3 \rho_{1}+\rho_{2}\right)^{3} h_{n s}+2 \rho_{2} a_{20}^{2}}{2 \operatorname{Im}(\lambda)}, \quad g_{u v}=-2\left(3 \rho_{1}+\rho_{2}\right)^{2} h_{n s}, \\
& g_{v v}=-2 h_{n s}\left(3 \rho_{1}+\rho_{2}\right) \operatorname{Im}(\lambda), \\
& f_{u v v}=f_{v v v}=f_{u u v}=f_{v v v}=g_{u u u}=g_{u u v}=g_{u v v}=g_{v v v}=0 .
\end{aligned}
$$

In order to undergo Neimark-Sacker bifurcation for map (2), the critical discriminatory quantity is not zero $[13,34]$ :

$$
\vartheta=\left.\left[-\operatorname{Re}\left(\frac{(1-2 \lambda) \bar{\lambda}^{2}}{1-\lambda} \xi_{20} \xi_{11}\right)-\frac{1}{2}\left|\xi_{11}\right|^{2}-\left|\xi_{02}\right|^{2}\right]\right|_{\bar{\mu}=0},
$$

where

$$
\xi_{20}=\frac{1}{8}\left[f_{u u}-f_{v v}+2 g_{u v}+i\left(g_{u u}-g_{v v}-2 f_{u v}\right)\right],
$$




$$
\begin{aligned}
& \xi_{11}=\frac{1}{4}\left[f_{u u}+f_{v v}+i\left(g_{u u}+g_{v v}\right)\right], \\
& \xi_{02}=\frac{1}{8}\left[f_{u u}-f_{v v}-2 g_{u v}+i\left(g_{u u}-g_{v v}+2 f_{u v}\right)\right] .
\end{aligned}
$$

From the above analysis and the theorems in $[13,34]$, we get the following results.

Theorem 3.1 If $\mu=\mu_{22}$ and $\vartheta \neq 0$, then map (2) undergoes a Neimark-Sacker bifurcation at $E_{2}\left(x_{2}, y_{2}\right)$ when $\mu$ varies in the neighbourhood of $\mu_{22}$. Moreover, if $\vartheta<0$ (resp., $\left.\vartheta>0\right)$, then an attracting (resp., repelling) invariant closed circle bifurcates from the fixed point for $\mu>\mu_{22}$ (resp., $\left.\mu<\mu_{22}\right)$.

In the section of numerical simulations, some parameter values are chosen to show the complex dynamics derived from the occurrence of Neimark-Sacker bifurcation. To understand the dynamics of Kopel oligopoly model fully, some two-parameter bifurcation analysis is introduced as follows.

\section{1:3 resonance for $E_{2}$}

In order to analyse the degenerate case of Neimark-Sacker bifurcation at $E_{2}$, another parameter should be considered. Without loss of generality, we choose $\rho_{1}$ as one of bifurcation parameters. Similarly, the following results about $\rho_{1}$ can be gotten with respect to $\rho_{2}$. In this section, we choose $\rho_{1}$ and $\mu$ as bifurcation parameters to present 1:3 resonance analysis at $E_{2}\left(x_{2}, y_{2}\right)$. In the rest of the paper, inner product method is introduced to simplify the transformation for normal form of different types of bifurcation.

Taking bifurcation parameters $\left(\rho_{1}, \rho_{2}, \mu_{2}\right)$ arbitrarily from $\left(\rho_{10}, \rho_{2}, \mu_{2}\right)$, we consider map (2) with $\left(\rho_{10}, \rho_{2}, \mu_{2}\right)$, which can be written as the following map:

$$
\left(\begin{array}{l}
x \\
y
\end{array}\right) \mapsto\left(\begin{array}{c}
\left(1-\rho_{10}\right) x+\rho_{10} \mu_{22} y(1-y) \\
\left(1-\rho_{2}\right) y+\rho_{2} \mu_{22} x(1-x)
\end{array}\right) .
$$

There exist the eigenvalues $\lambda_{1,2}=\frac{ \pm \sqrt{3} i-1}{2}$, at $E_{2}\left(x_{2}, y_{2}\right)$ of map (10).

Now, we consider the perturbation map as follows:

$$
\left(\begin{array}{l}
x \\
y
\end{array}\right) \mapsto\left(\begin{array}{c}
\left(1-\rho_{1}\right) x+\rho_{1} \mu y(1-y) \\
\left(1-\rho_{2}\right) y+\rho_{2} \mu x(1-x)
\end{array}\right),
$$

where $\left|\rho_{1}-\rho_{10}\right|,\left|\mu-\mu_{22}\right| \ll 1$.

Let $u=x-x_{2}$ and $v=y-y_{2}$. Then we transform $E_{2}\left(x_{2}, y_{2}\right)$ to the origin $(0,0)$, and map (11) becomes

$$
\left(\begin{array}{l}
u \\
v
\end{array}\right) \mapsto\left(\begin{array}{c}
\left(1-\rho_{1}\right) u+\rho_{1}\left(1-2 y_{2}\right) \mu v-\rho_{1} \mu v^{2} \\
\left(1-\rho_{2}\right) v+\rho_{2}\left(1-2 x_{2}\right) \mu u-\rho_{2} \mu u^{2}
\end{array}\right) .
$$

In the following, we introduce coordinates transformation and the inner product method to present our analysis in the critical case. Let $b_{20}=\rho_{10}\left(1-2 y_{2}\right) \mu_{22}$ and use the following transformation:

$$
\left(\begin{array}{l}
u \\
v
\end{array}\right)=\left(\begin{array}{cc}
-b_{20} & 0 \\
\frac{3}{2}-\rho_{10} & -\frac{\sqrt{3}}{2}
\end{array}\right)\left(\begin{array}{c}
\tilde{x} \\
\tilde{y}
\end{array}\right)
$$


for (12); then map (12) will be transformed to that as follows:

$$
\begin{aligned}
\left(\begin{array}{l}
\tilde{x} \\
\tilde{y}
\end{array}\right) \mapsto & \left(\begin{array}{cc}
-\frac{1}{2} & \frac{\sqrt{3}}{2} \\
-\frac{\sqrt{3}}{2} & -\frac{1}{2}
\end{array}\right)\left(\begin{array}{l}
\tilde{x} \\
\tilde{y}
\end{array}\right) \\
& +\frac{\rho_{10} \mu_{22}}{12 b_{20}}\left(\begin{array}{c}
\left.3\left[\left(3-2 \rho_{10}\right) \tilde{x}-\sqrt{3} \tilde{y}\right)\right]^{2} \\
\left.\sqrt{3}\left(3-2 \rho_{10}\right)\left[\left(3-2 \rho_{10}\right) \tilde{x}-\sqrt{3} \tilde{y}\right)\right]^{2}+\frac{8 \sqrt{3}}{\rho_{10}} \rho_{2} \mu_{22} b_{20}^{3} \tilde{x}^{2}
\end{array}\right) .
\end{aligned}
$$

The eigenvalues of linearization matrix of map (13) are $\frac{ \pm \sqrt{3} i-1}{2}$ and their corresponding eigenvector $q\left(\rho_{10}, \mu_{22}\right)=(1, i) \in \mathbb{C}^{2}$. Furthermore, the adjoint eigenvector $p\left(\rho_{10}, \mu_{22}\right)=$ $\left(\frac{1}{2}, \frac{i}{2}\right) \in \mathbb{C}^{2}$, satisfying $\left\langle p\left(\rho_{10}, \mu_{22}\right), q\left(\rho_{10}, \mu_{22}\right)\right\rangle=1$, where $\langle p, q\rangle=\bar{p}_{1} q_{1}+\bar{p}_{2} q_{2}$.

Now arbitrary vector $x \in \mathbb{R}^{2}$ can be decomposed as the following form:

$$
x=z q\left(\rho_{10}, \mu_{22}\right)+\bar{z} \overline{q\left(\rho_{10}, \mu_{22}\right)},
$$

and map (13) can be rewritten as

$$
z \longmapsto \frac{\sqrt{3} i-1}{2} z+\sum_{j+k=2} \frac{1}{j ! k !} g_{j k}\left(\rho_{10}, \mu_{22}\right) z^{j} \bar{z}^{k}
$$

where

$$
\begin{aligned}
& g_{20}=\left[3\left(3-2 \rho_{10}\right)^{2}-\sqrt{3}\left(3-2 \rho_{10}\right)^{3} i-\frac{8 \sqrt{3} i}{\rho_{10}} \rho_{2} \mu_{22} b_{20}^{3}\right] h, \\
& g_{11}=3 h\left(\left(3-2 \rho_{10}\right) i-\sqrt{3}\right)\left(3-2 \rho_{10}\right), \\
& g_{02}=3 h\left(3-\sqrt{3}\left(3-2 \rho_{10}\right) i\right), \\
& h=\frac{\rho_{10} \mu_{22}}{4 b_{20}} .
\end{aligned}
$$

Here, we denote $g_{j k}\left(\rho_{11}, \mu_{22}\right)$ with $j+k=2$ by $g_{j k}$ with $j+k=2$ for simplicity.

According to transformation analysis as in [20], map (14) finally becomes the following normal form of 1:3 resonance:

$$
\zeta \mapsto \frac{\sqrt{3} i-1}{2} \zeta+B\left(\rho_{10}, \mu_{22}\right) \bar{\zeta}^{2}+C\left(\rho_{10}, \mu_{22}\right) \zeta \bar{\zeta}^{2}+O\left(|\bar{\zeta}|^{4}\right)
$$

where

$$
\begin{aligned}
& B\left(\rho_{10}, \mu_{22}\right)=\frac{g_{02}}{2}, \\
& C\left(\rho_{10}, \mu_{22}\right)=\frac{g_{20} g_{11}(3+2 \sqrt{3} i)}{6}+\frac{(3-\sqrt{3} i)\left|g_{11}\right|^{2}}{6} .
\end{aligned}
$$

If $B_{1}\left(\rho_{10}, \mu_{22}\right)=-\frac{3}{2}(\sqrt{3} i+1) B\left(\rho_{10}, \mu_{22}\right), C_{1}\left(\rho_{10}, \mu_{22}\right)=-3\left|B\left(\rho_{10}, \mu_{22}\right)\right|^{2}-\frac{3(1+\sqrt{3} i)}{2} C\left(\rho_{10}\right.$, $\left.\mu_{22}\right)$, a similar argument as in Lemma 9.13 in [19] can be obtained.

Theorem 4.1 If $B_{1}\left(\rho_{10}, \mu_{22}\right) \operatorname{Re}\left(C_{1}\left(\rho_{10}, \mu_{22}\right)\right) \neq 0$, then there exist the following local and global dynamics in the neighbourhood of $E_{2}$ for map (15): 
(a) There exists a Neimark-Sacker bifurcation curve at the origin of map (15).

(b) There exists a saddle cycle of period-3 corresponding to three saddle fixed points of map (15).

(c) There exists a homoclinic structure induced by the above period-3 cycle.

In Sect. 6, some parameter values are chosen to show the occurrence of 1:3 resonance bifurcation. Local and global dynamics in the neighbourhood of 1:3 resonant point are illustrated in a two-parameter plane.

\section{1:4 resonance for $E_{2}$}

In this section, specific conditions for parameters derived to show that map (2) may undergo 1:4 strong resonance [13, 34]. Taking parameters $\left(\rho_{1}, \rho_{2}, \mu\right)$ arbitrarily from $\left(\rho_{11}, \rho_{2}, \mu_{22}\right)$, we consider map (2) with $\left(\rho_{11}, \rho_{2}, \mu_{22}\right)$, which is described by

$$
\left(\begin{array}{l}
x \\
y
\end{array}\right) \mapsto\left(\begin{array}{c}
\left(1-\rho_{11}\right) x+\rho_{11} \mu_{22} y(1-y) \\
\left(1-\rho_{2}\right) y+\rho_{2} \mu_{22} x(1-x)
\end{array}\right),
$$

and the eigenvalues of map (16) at $E_{2}\left(x_{2}, y_{2}\right)$ are $\lambda_{1,2}= \pm i$.

Let $u=x-x_{2}$ and $v=y-y_{2}$, without confusion. Then we get the following map at the origin, map (16) becomes

$$
\left(\begin{array}{l}
u \\
v
\end{array}\right) \mapsto\left(\begin{array}{c}
\left(1-\rho_{11}\right) u+c_{20} v-\rho_{11} \mu_{22} v^{2} \\
\left(1-\rho_{2}\right) v+c_{21} u-\rho_{2} \mu_{22} u^{2}
\end{array}\right),
$$

where

$$
\begin{aligned}
& c_{20}=\rho_{11}\left(1-2 y_{2}\right) \mu_{22}, \\
& c_{21}=\rho_{2}\left(1-2 x_{2}\right) \mu_{22} .
\end{aligned}
$$

Let

$$
\left(\begin{array}{l}
u \\
v
\end{array}\right)=\left(\begin{array}{cc}
-c_{20} & 0 \\
1-\rho_{11} & -1
\end{array}\right)\left(\begin{array}{c}
\tilde{x} \\
\tilde{y}
\end{array}\right)
$$

then map (17) becomes

$$
\left(\begin{array}{c}
\tilde{x} \\
\tilde{y}
\end{array}\right) \mapsto\left(\begin{array}{cc}
0 & -1 \\
1 & 0
\end{array}\right)\left(\begin{array}{c}
\tilde{x} \\
\tilde{y}
\end{array}\right)+\frac{\rho_{11} \mu_{2}}{c_{20}}\left(\begin{array}{c}
\left.\left[\left(1-\rho_{11}\right) \tilde{x}-\tilde{y}\right)\right]^{2} \\
\left.\left[\left(1-\rho_{11}\right) \tilde{x}-\tilde{y}\right)\right]^{2}-\frac{\rho_{2} b_{20}^{3}}{\rho_{11}} \tilde{x}^{2}
\end{array}\right) .
$$

We have that the Jacobian matrix of map (18) at the origin has the eigenvalues $\lambda_{1,2}=$ $\pm i$ and eigenvectors $q_{1,2}=(1, \mp i)$, respectively. As above, any vector $(x, y)^{T} \in R^{2}$ can be rewritten as the complex form $(x, y)^{T}=z q+\overline{z q}$.

Then map (18) can be transformed to the complex form as follows:

$$
z \mapsto i z+G\left(z, \bar{z}, \rho_{11}, \mu_{22}\right),
$$


where

$$
G\left(z, \bar{z}, \rho_{11}, \mu_{22}\right)=\sum_{k+l=2} \frac{1}{k ! l !} \breve{g}_{k l} z^{k} \bar{z}^{l}
$$

and

$$
\begin{aligned}
& \breve{g}_{20}=-\frac{c_{20}^{2} \mu_{22} \rho_{2} i}{2}+\frac{\mu_{22} \rho_{11}\left(\rho_{11}-1-i\right)^{2}(1+i)}{2 c_{20}}, \\
& \breve{g}_{11}=-c_{20}^{2} \mu_{22} \rho_{2} i+\frac{\mu_{22} \rho_{11}\left(\rho_{11}-1-i\right)\left(\rho_{11}-1+i\right)(1+i)}{c_{20}}, \\
& \breve{g}_{02}=-\frac{c_{20}^{2} \mu_{22} \rho_{2} i}{2}+\frac{\mu_{22} \rho_{11}\left(\rho_{11}-1+i\right)^{2}(1+i)}{2 c_{20}} .
\end{aligned}
$$

Here, we denote $\breve{g}_{i j}\left(\rho_{11}, \mu_{22}\right)$ with $i+j=2,3$ by $\breve{g}_{i j}$ with $i+j=2,3$ for simplicity, too.

According to transformation analysis as in [22], map (19) can be finally transformed to the following form:

$$
\zeta \mapsto i \zeta+\varrho_{21}\left(\rho_{11}, \mu_{22}\right) \zeta^{2} \bar{\zeta}+\varrho_{03}\left(\rho_{11}, \mu_{22}\right) \zeta^{3}+O\left((|\zeta|+|\bar{\zeta}|)^{4}\right)
$$

where

$$
\begin{aligned}
& \varrho_{21}\left(\rho_{11}, \mu_{22}\right)=\frac{1+3 i}{4} \breve{g}_{20} \breve{g}_{11}+\frac{1-i}{2}\left|\breve{g}_{11}\right|^{2}-\frac{1+i}{4}\left|\breve{g}_{02}\right|^{2}, \\
& \varrho_{03}\left(\rho_{11}, \mu_{22}\right)=\frac{i-1}{4} \breve{g}_{02} \breve{g}_{11}-\frac{1+i}{4} \breve{g}_{11} \breve{g}_{20} .
\end{aligned}
$$

Let $C_{1}\left(\rho_{11}, \mu_{22}\right)=-4 i \varrho_{21}\left(\rho_{11}, \mu_{22}\right), D_{1}\left(\rho_{11}, \mu_{22}\right)=-4 i \varrho_{03}\left(\rho_{11}, \mu_{22}\right)$. If $D_{1}\left(\rho_{11}, \mu_{22}\right) \neq 0$, we denote $A=\frac{C_{1}\left(\rho_{11}, \mu_{22}\right)}{\left|D_{1}\left(\rho_{11}, \mu_{22}\right)\right|}$. By a similar argument as in Lemma 9.15 in [19], we can obtain the following result.

Theorem 5.1 If $D_{1}\left(\rho_{11}, \mu_{22}\right) \neq 0, \operatorname{Re} A\left(\rho_{11}, \mu_{22}\right) \neq 0$ and $\operatorname{Im} A\left(\rho_{11}, \mu_{22}\right) \neq 0$, then map (20) contains four different types of bifurcation curves as follows:

(a) There exists a Neimark-Sacker bifurcation curve at the origin of map (20).

(b) There exist bifurcation curves of saddle-node and Neimark-Sacker at eight nontrivial fixed points of map (20).

(c) There is a "square" heteroclinic cycle around the origin of map (20).

\section{Numerical analysis}

With the help of MATLAB, the critical coefficients of three kinds of bifurcations are computed and different kinds of figures are plotted for map (2) in this section.

When $\rho_{1}=0.3, \rho_{2}=0.8, \mu=1+\frac{\sqrt{1236}}{12}$, we obtain a nontrivial positive fixed point $(0.899629235032409,0.354841003016974)$ for map (2) from the third case of Lemma 2.1. When $\mu=1+\frac{\sqrt{1236}}{12}$, Neimark-Sacker bifurcation occurs at $(0.899629235032409$, $0.354841003016974)$ and its eigenvalues are $\lambda_{1,2}=\frac{9}{20} \pm \frac{\sqrt{319}}{20} i$. Hence, there are $|\lambda|=1$, $l=\frac{d|\lambda|}{d \mu}=-0.55>0$ and $\vartheta=-9.7875$.

From Figs. 2(a)-(d), complex dynamics are observed, such as fixed points, multi-period orbits, (multiple) invariant closed orbits and chaotic orbits. See Fig. 3 for more informa- 


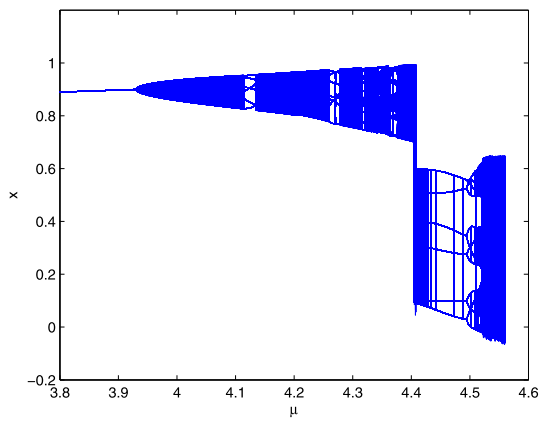

(a)

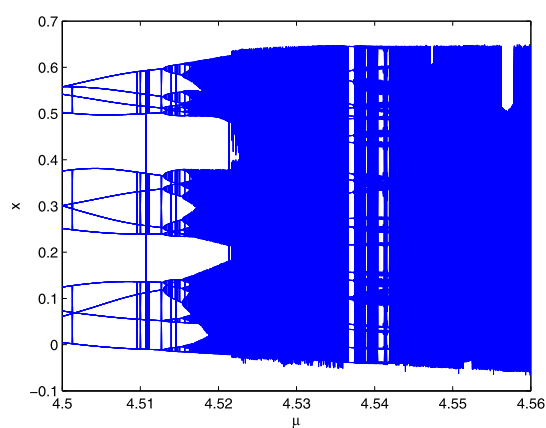

(c)

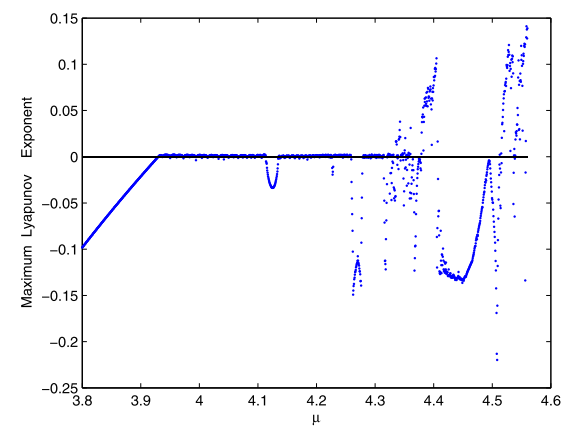

(e)

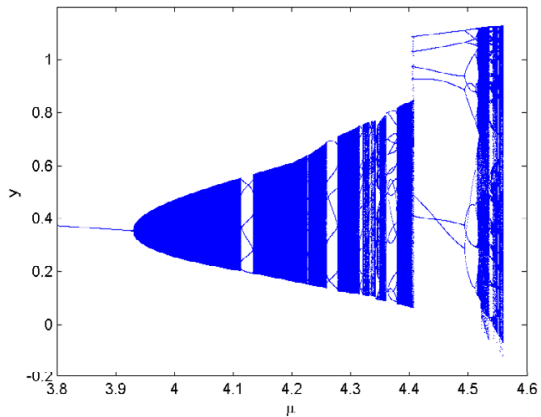

(b)

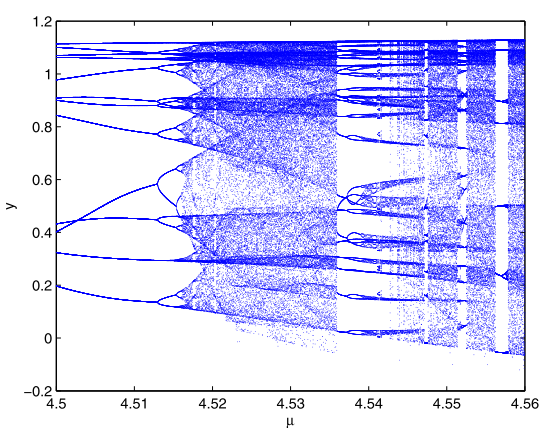

(d)

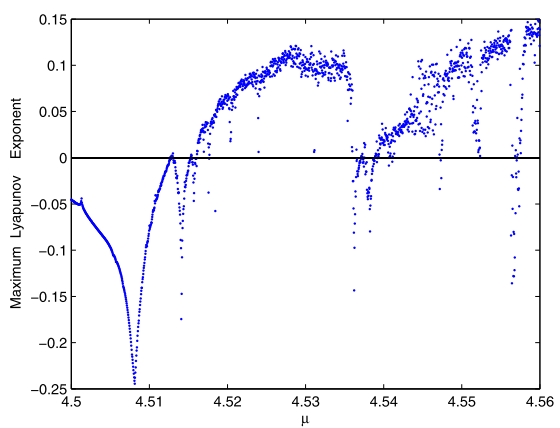

(f)

Figure 2 (a) Bifurcation diagram of map (2) in $(\mu, x)$ plane for $\rho_{1}=0.3, \rho_{2}=0.8$ and $\mu \in[3.8,4.6]$. The initial point is $(0.899,0.354)$. (b) Bifurcation diagram of map (2) in $(\mu, x)$ plane corresponding to (a). (c) Local amplification corresponding to (a) for $\mu \in$ [4.54.56]. (d) Local amplification corresponding to (b) for $\mu \in[4.5,4.56]$. (e) Maximum Lyapunov exponents (MLE for short) corresponding to (a) and (b). (f) Local amplification corresponding to (e)

tion. Especially, there exist "period bubbling" phenomena [33] when $\mu \in(4.4,4.517) \cup$ $(4.537,4.543)$ in Figs. $2(\mathrm{c})-(\mathrm{d})$. To show the stability clearly, we computed the maximum Lyapunov exponents (MLE for short) and plotted them in Figs. 2(e) and (f). The selected phase portraits are displayed in Fig. 3, which illustrates the evolving process of closed invariant circle. When $\mu=4.347186$, there exist a series of "small" closed orbits. When $\mu=4.4386$, the closed invariant circle changes to a period-6 orbit, and the "period bubbling" phenomenon occurs. 


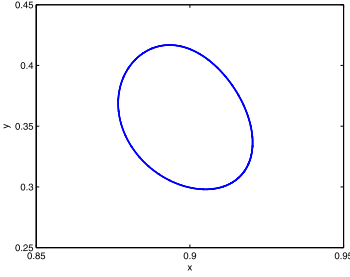

(a)

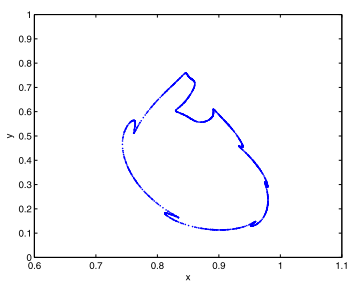

(d)

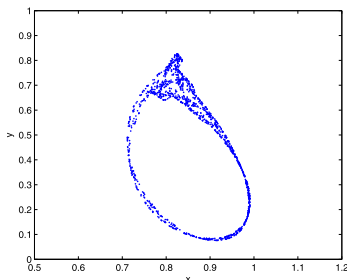

(g)

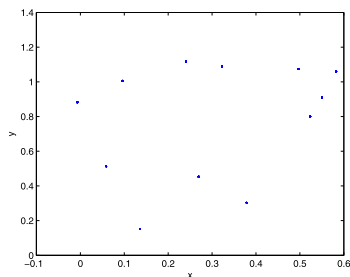

(j)

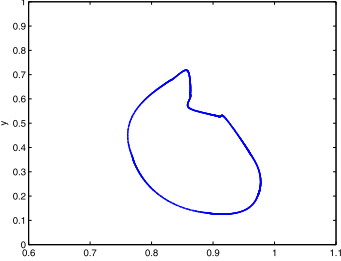

(b)

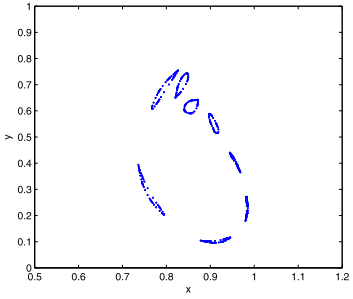

(e)

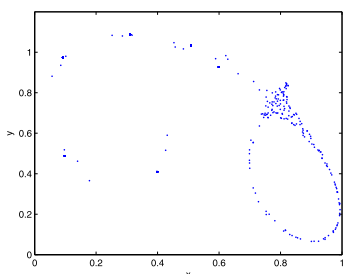

(h)

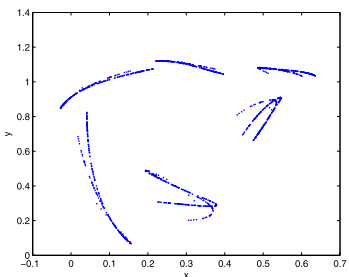

(k)

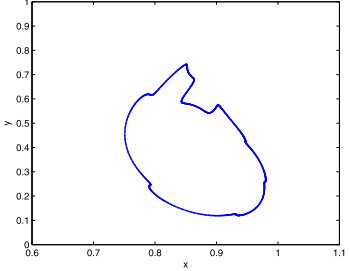

(c)

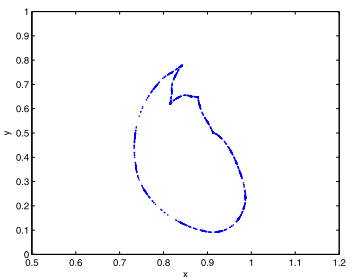

(f)

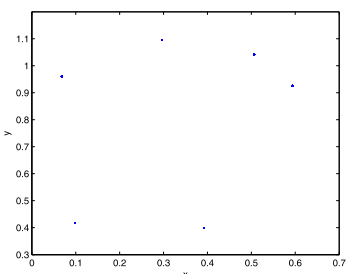

(i)

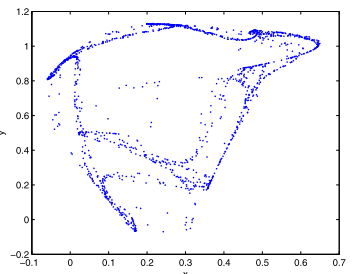

(1)

Figure 3 Phase portraits for different values of $\mu$. (a) $\mu=3.9517$; (b) $\mu=4.2863$; (c) $\mu=4.3091$; (d) $\mu=4.3243$; (e) $\mu=4.3471$; (f) $\mu=4.3851$; (g) $\mu=4.4079$; (h) $\mu=4.4383$; (i) $\mu=4.3547$; (j) $\mu=4.5068$; (k) $\mu=4.5220$; (I) $\mu=4.5600$

When $\rho_{1}=1.2, \rho_{2}=1.8, \mu=1+\frac{\sqrt{194}}{6}$, we get a nontrivial positive fixed point $(0.827950951929977,0.473127083912457)$ for map (2) from the third case of Lemma 2.1 . After computation, we obtain that the eigenvalues of Jacobian matrix of map (2) are $\lambda_{1,2}=\frac{-1 \pm \sqrt{3} i}{2}$ with $B_{1}=-50.2365-43.506 i \neq 0$ and $C_{1}=-1653.3-27.329 i \neq 0$. There exist $1: 3$ resonance phenomena emerging from $(0.827950951929977,0.473127083912457)$.

When $\rho_{1}=1.2, \rho_{2}=1.8$, Fig. 4(a) shows the 2D bifurcation diagrams with the change of $\mu$. As the local amplification of 4(a), 4(b) is presented to show the occurrence of 1:3 resonance accurately. Corresponding MLE are illustrated in Fig. 4(c), and the red points are computed when $\mu=1+\frac{\sqrt{194}}{6}$. When $\rho_{1}=1.5, \rho_{2}=1.8$, different dynamics appear, such as the period-3 closed orbits, see Fig. 4(d). Related numerical results are showed through the following analysis of phase diagrams in Fig. 5. 


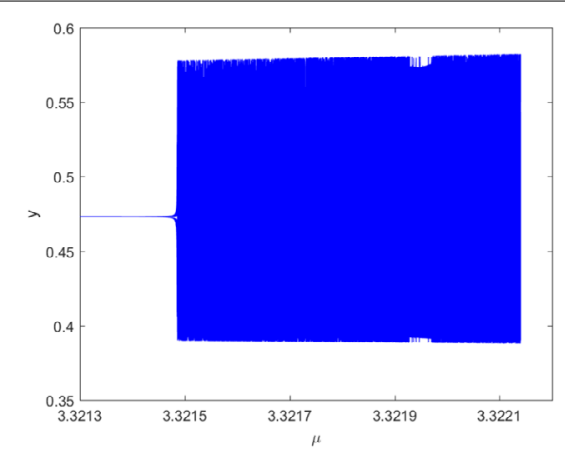

(a)

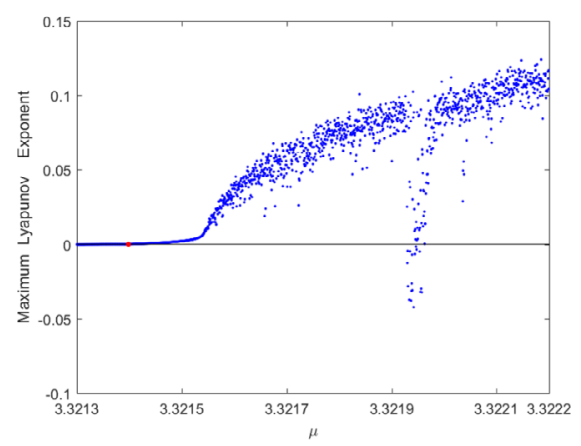

(c)

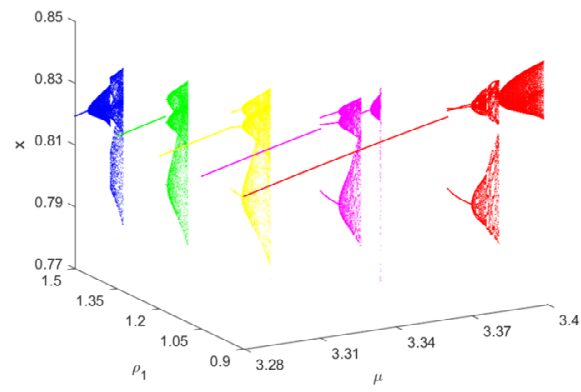

(e)

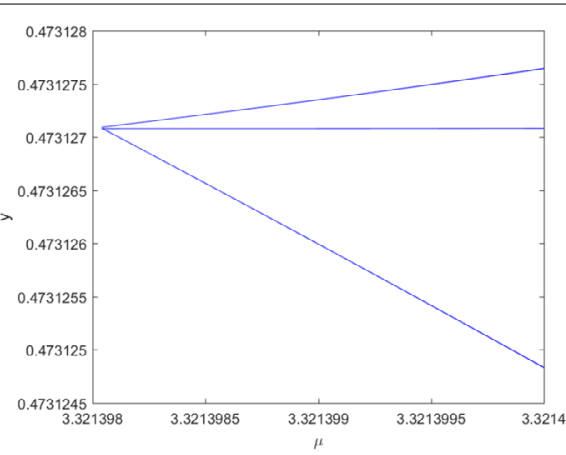

(b)

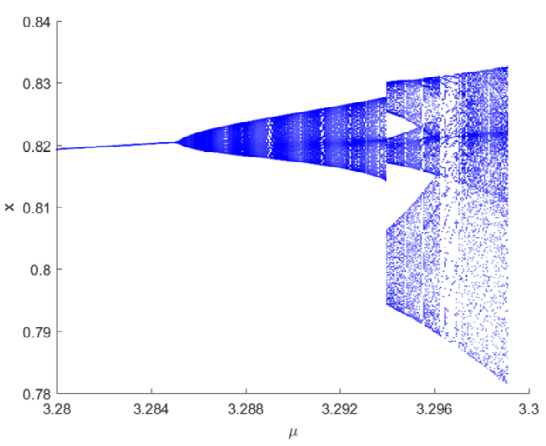

(d)

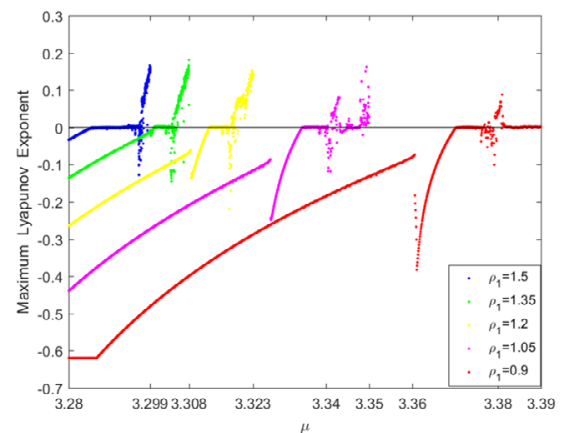

(f)

Figure 4 (a) Bifurcation diagram of map (2) in $(\mu, y)$ space for $\rho_{1}=1.2, \rho_{2}=1.8$, the initial point is (0.82795095, 0.47312708). (b) Local amplification for $\mu \in[3.321398,3.3214]$ (c) MLE corresponding to (a). (d) Bifurcation diagram of map (2) in $(\mu, x)$ space for $\rho_{1}=1.5, \rho_{2}=1.8$ the initial point is $(0.82,0.47)$. (e) Bifurcation diagram of map (2) in $\left(\mu, \rho_{1}, x\right)$ space for $\rho_{2}=1.8$. (f) MLE corresponding to (e)

When $\rho_{2}=1.8$ and $\left(\rho_{1}, \mu\right)$ varies near $(1.2,3.3214)$, 3D bifurcation diagrams for map (2) are displayed in Fig. 4(e). Because of the sensitive dependence on initial points for map (2), 3D figures would not be provided as in the following case of 1:4 resonance. The corresponding MLE presented in Fig. 4(f) shows the complexity of dynamics clearly, such as the onset of chaos and periodic orbits. We observe that the stable region of corresponding fixed points shrinks when $\rho_{1}$ increases. From Fig. 5(a), we can see that $(0.827950951929977,0.473127083912457)$ becomes three fixed points through 1:3 resonance, and these three fixed points evolve to three stable invariant circles plotted in Fig. 5(b), which eventually lead to chaotic orbits. When $\rho_{1}=1.05, \mu=3.3486$, there exists a saddle cycle of period-3 in Fig. 5(k). Furthermore, the corresponding saddle fixed points 


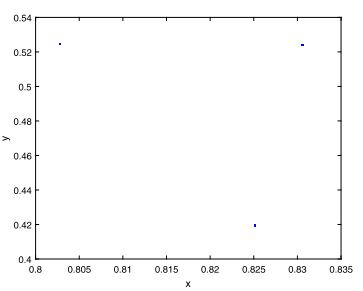

(a)

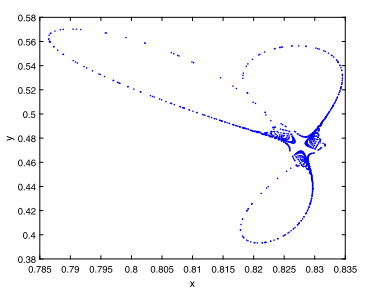

(d)

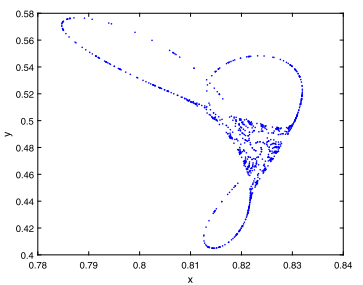

(g)

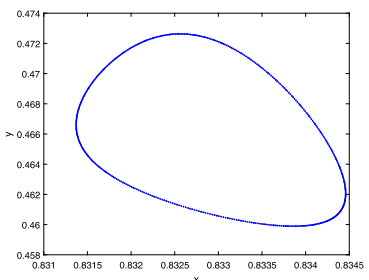

(j)

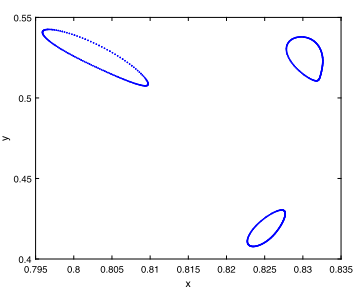

(b)

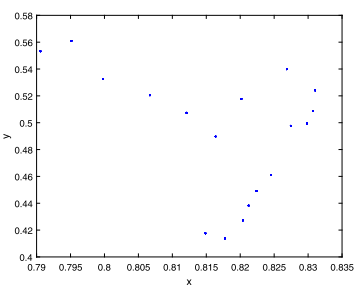

(e)

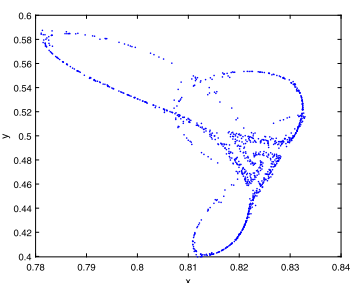

(h)

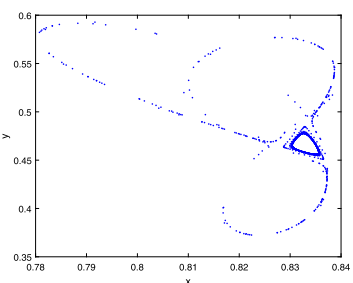

(k)

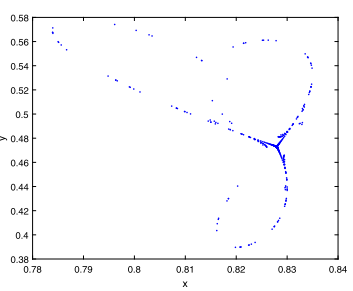

(c)

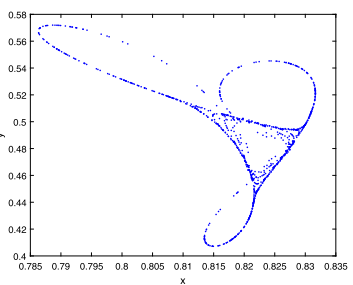

(f)

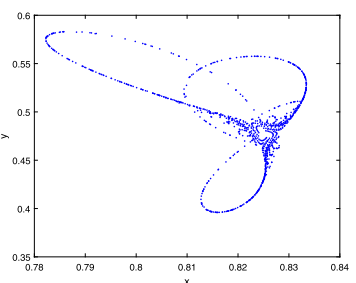

(i)

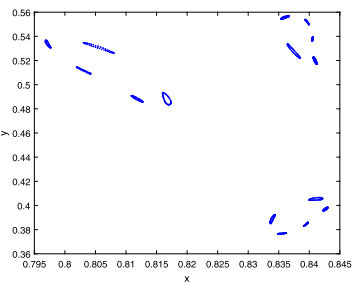

(1)

Figure 5 Phase portraits for different values of $\left(\rho_{1}, \mu\right)$. (a) $\rho_{1}=1.2, \mu=3.3118$; (b) $\rho_{1}=1.2, \mu=3.3148$; (c) $\rho_{1}=1.2, \mu=3.321495 ;$ (d) $\rho_{1}=1.2, \mu=3.3202$; (e) $\rho_{1}=1.5, \mu=3.2964 ;(\mathbf{f}) \rho_{1}=1.5, \mu=3.2974$; (g) $\rho_{1}=1.5, \mu=3.2980$; (h) $\rho_{1}=1.5, \mu=3.2990$; (i) $\rho_{1}=1.35, \mu=3.3073$; (j) $\rho_{1}=1.05, \mu=3.3475$; (k) $\rho_{1}=1.05, \mu=3.3486$; (I) $\rho_{1}=0.9, \mu=3.3768$

are three vertices of the triangle. In fact, a series of stable closed orbits are observed in Fig. 5 when $\rho_{1}=0.9, \mu=3.3768$. Homoclinic structure is illustrated in Fig. 5 , which means that different evolve direction leads to the same results.

When $\rho_{1}=1.4, \rho_{2}=0.6, \mu=1+\frac{\sqrt{2814}}{21}$, we get a positive fixed point $(0.860606075514091$, 0.422997011561125 ) for map (2) from the third case of Lemma 2.1. After computation, we obtain that the eigenvalues of Jacobian matrix of map (2) are $\lambda_{1,2}= \pm i$ when $\rho_{1}=1.4$, $\rho_{2}=0.6$, and $\mu=1+\frac{\sqrt{2814}}{21}$ with $C_{1}=-158.09-337.25 i \neq 0$ and $D_{1}=72.9470-91.0586 i \neq 0$. When $\rho_{1}=1.4, \rho_{2}=0.6$, Fig. $6(\mathrm{a})$ is $2 \mathrm{D}$ bifurcation diagrams with the change of $\mu$. When $\rho_{2}=0.6$ and $\rho_{1}, \mu$ vary near $(1.4,3.5260)$, 3D bifurcation diagrams for map (2) are presented in Fig. 6(c). The corresponding MLE are presented in Figs. 6(b) and (d) to explore the different kinds of stability at the fixed points of map (2). Moreover, 3D figures are pro- 


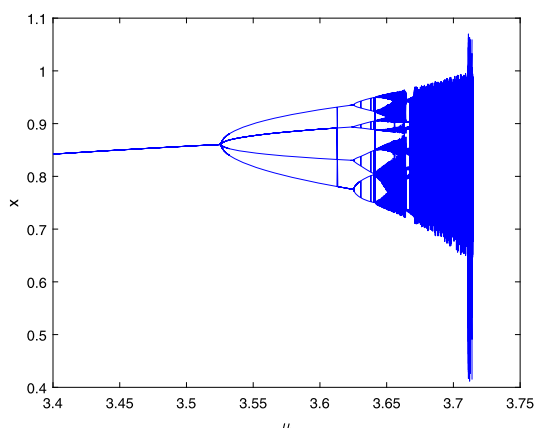

(a)

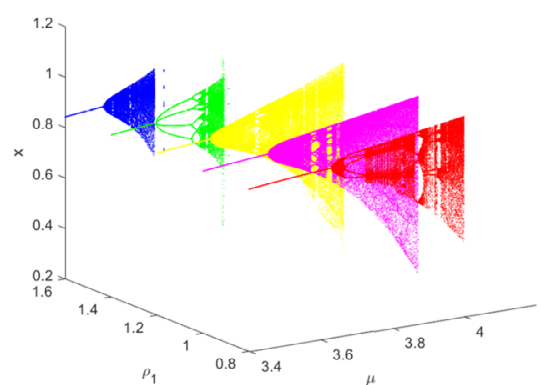

(c)

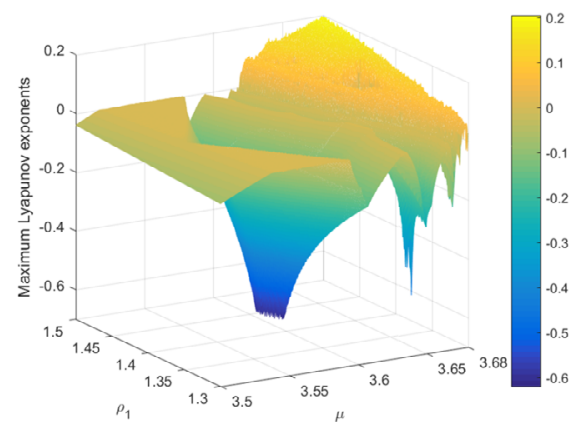

(e)

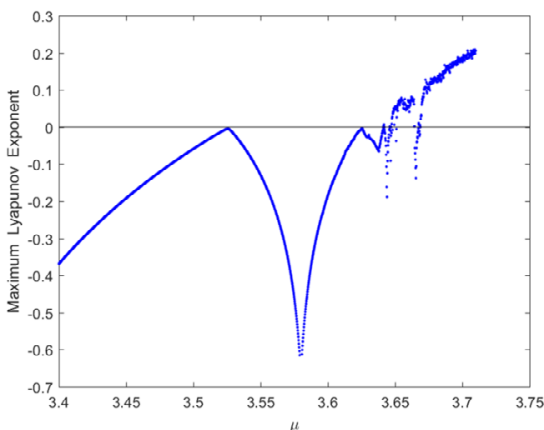

(b)

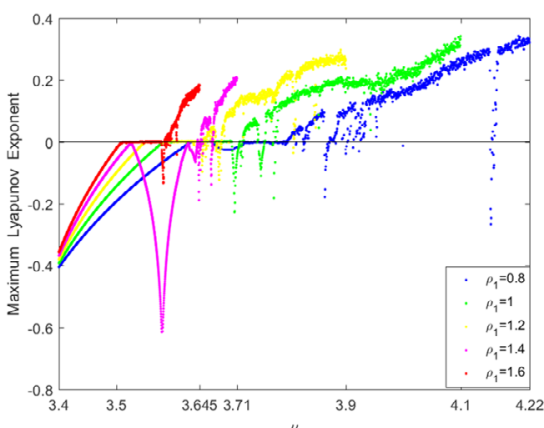

(d)

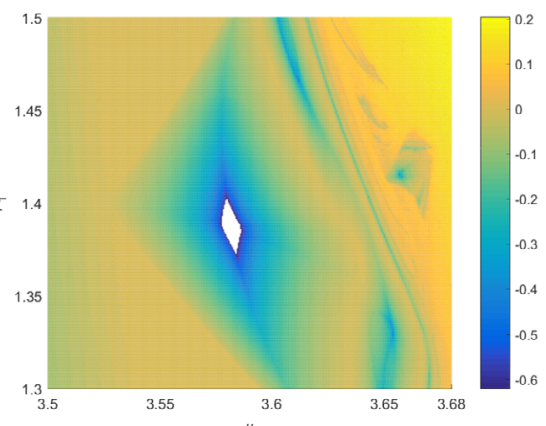

(f)

Figure 6 (a) Bifurcation diagram of map (2) in $(\mu, x)$ space for $\rho_{1}=1.4, \rho_{2}=0.6$ the initial value is $(0.87,0.4)$. (b) MLE corresponding to (a). (c) Bifurcation diagram of map (2) in $\left(\mu, \rho_{1}, x\right)$ space for $\rho_{2}=0.6$ (d) MLE corresponding to (b). (e) MLE corresponding to (b). (f) MLE corresponding to (e)

vided to show different kinds of stability when $\left(\rho_{1}, \mu\right) \in[1.3,1.5] \times[3.55,3.67]$. White hole in Fig. 6(f) implies that MLE are smaller than 0.6, and orbits emerging from the hole will converge to the corresponding fixed point. See related phase portraits are plotted in Fig. 7 for more information.

Figure 7 shows the phase portraits of map (2) induced by 1:4 resonance. From Fig. 7(a)(g), $(0.860606075514091,0.422997011561125)$ becomes period- $4 n(n=1,2,4,8)$ and eventually becomes different shapes of a chaotic set, such as the shape of boots. In fact, further investigation reveals that it is impossible for the points inside the chaotic region to escape, see Fig. 7(h). Different types of strange attractors are presented to illustrate complex dynamics in Fig. 7(i)-(l). 


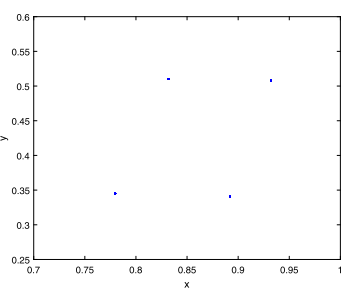

(a)

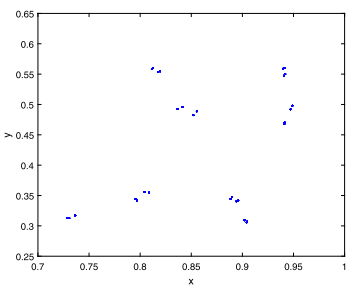

(d)

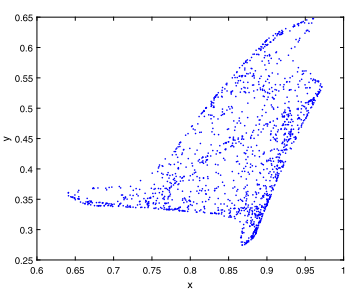

(g)

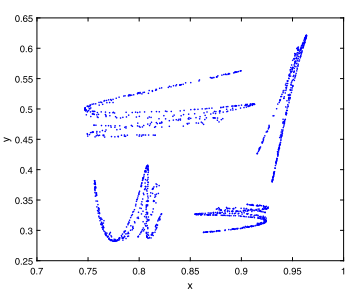

(j)

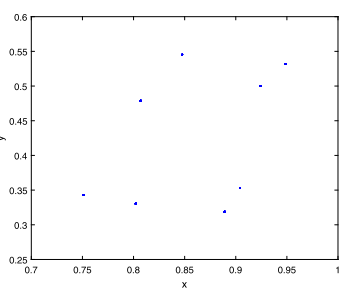

(b)

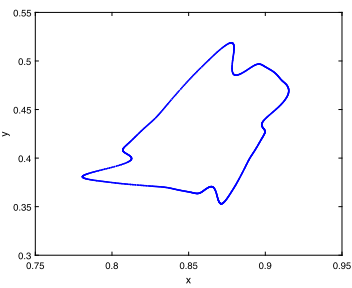

(e)

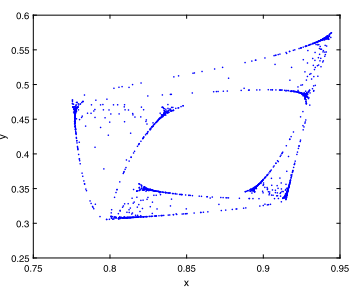

(h)

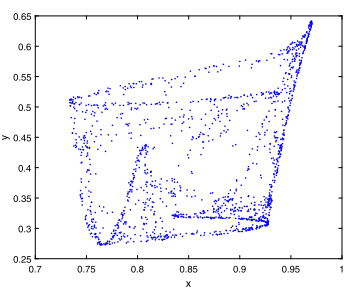

(k)

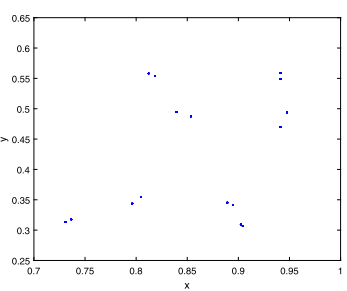

(c)

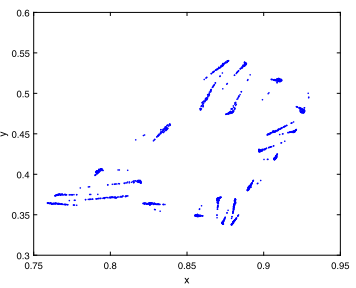

(f)

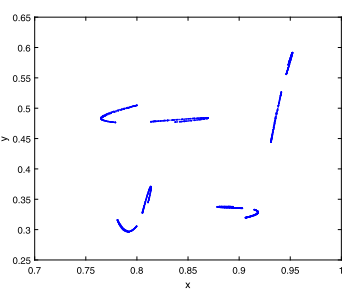

(i)

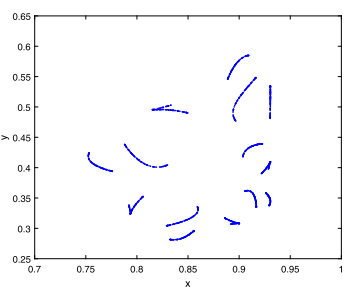

(1)

Figure 7 Phase portraits for various values of $\left(\rho_{1}, \mu\right)$ corresponding to Fig. 6. (a) $\rho_{1}=1.4, \mu=3.615$; (b) $\rho_{1}=1.4, \mu=3.639 ;$ (c) $\rho_{1}=1.4, \mu=3.667 ;$ (d) $\rho_{1}=1.4, \mu=3.668 ;$ (e) $\rho_{1}=1.6, \mu=3.5673 ;$ (f) $\rho_{1}=1.6$, $\mu=3.5948 ;(\mathbf{g}) \rho_{1}=1.6, \mu=3.6448$; (h) $\rho_{1}=1.2, \mu=3.6723$; (i) $\rho_{1}=1.2, \mu=3.6873$; (j) $\rho_{1}=1.2, \mu=3.7099$; (k) $\rho_{1}=1.2, \mu=3.7249 ;$ (I) $\rho_{1}=1, \mu=3.7224$

\section{Conclusion}

In this research, we investigated complex dynamics of map (2) and concluded that Kopel oligopoly model could undergo Neimark-Sacker bifurcation and the general cases, that is, 1:3 and 1:4 resonances. The critical normal form of bifurcation was introduced to carry out bifurcation analysis for map (2). Complex dynamics observed from the presented phase portraits implied that outputs of two oligopolists can oscillate in the multiple-period and quasi-period or chaotic orbits.

Some symmetric phenomena and global orbits are observed in the case of 1:3 and 1:4 resonances. It is important to note that the symmetry illustrated by phase portraits is topologically equivalent to that for the normal form of $1: 3$ resonance. The occurrence of homoclinic orbits makes us believe that different ways and speeds of response may lead to 
the same state of oligopolist competition between two rivals. Map (2) is invariant under the rotation $P\left(P^{4}=I_{2}\right)$ through the angle $\frac{\pi}{2}$ because of 1:4 resonance. In future, the cases of different responses between oligopolists should be considered to explore the complex dynamics, such as different competitive strategies, timing of play. Based on extensive data collection and super computing power, empirical analysis will be potential research direction.

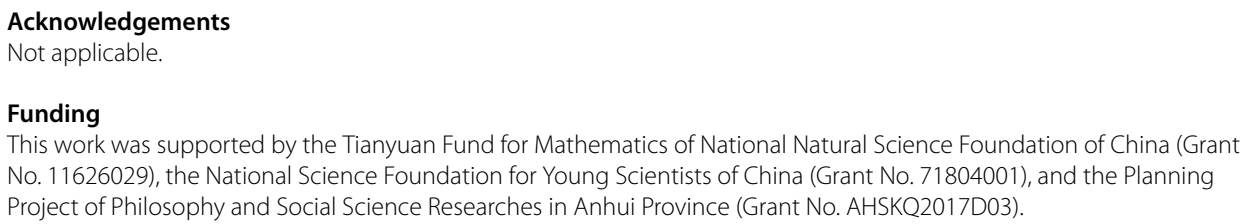

Availability of data and materials

Not applicable.

Competing interests

The authors declare that they have no competing interests.

Authors' contributions

All authors contributed equally in this article. All authors read and approved the final manuscript.

Authors' information

Not applicable.

\section{Publisher's Note}

Springer Nature remains neutral with regard to jurisdictional claims in published maps and institutional affiliations.

Received: 19 October 2019 Accepted: 6 February 2020 Published online: 18 February 2020

References

1. Agiza, H.N.: On the analysis of stability, bifurcation, chaos and chaos control of Kopel map. Chaos Solitons Fractals 10(11), 1906-1916 (1999)

2. Andaluz, J., Jarne, G.: Stability of vertically differentiated Cournot and Bertrand-type models when firms are boundedly rational. Ann. Oper. Res. 238, 1-25 (2016)

3. Anderson, D.R., Myran, N.G., White, D.L.: Basin of attraction in Cournot duopoly model of Kopel. J. Differ. Equ. Appl. 11(10), 879-887 (2005)

4. Baiardi, L.C., Naimzada, A.K.: An oligopoly model with best response and imitation rules. Appl. Math. Comput. 336 193-205 (2018)

5. Cánovas, J.S., Muñoz-Guillermo, M.: On the dynamics of Kopel's Cournot duopoly model. Appl. Math. Comput. 330, 292-306 (2018)

6. Colombo, L., Labrecciosa, P.: Consumer surplus-enhancing cooperation in a natural resource oligopoly. J. Environ. Econ. Manag. 92 185-193 (2018)

7. Colombo, L., Labrecciosa, P.: Stackelberg versus Cournot: a differential game approach. J. Econ. Dyn. Control 101, 239-261 (2019)

8. Cournot, A.: Researches into the Principles of the Theory of Wealth (Irwin Paper Back Classics in Economics). Hachette, Paris (1963) (in English)

9. Elsadany, A.A.: Dynamics of a Cournot duopoly game with bounded rationality based on relative profit maximization. Appl. Math. Comput. 294, 253-263 (2017)

10. Elsadany, A.A., Awad, A.M.: Dynamical analysis and chaos control in a heterogeneous Kopel duopoly game. Indian J. Pure Appl. Math. 47(4), 617-639 (2016)

11. Gao, X., Zhong, W.J., Mei, S.: Equilibrium stability of a nonlinear heterogeneous duopoly game with extrapolative foresight. Math. Comput. Simul. 8, 2069-2078 (2012)

12. Govaerts, W., Khoshsiar Ghaziani, R.: Stable cycles in a Cournot duopoly model of Kopel. J. Comput. Appl. Math. 218, 247-258 (2008)

13. Guckenheimer, J., Holmes, P.: Nonlinear Oscillations, Dynamical Systems, and Bifurcations of Vector Fields. Springer, Berlin (1983)

14. Hommes, C.H., Ochea, M.I., Tuinstra, J.: Evolutionary competition between adjustment processes in Cournot oligopoly: instability and complex dynamics. Dyn. Games Appl. 8, 822-843 (2018)

15. Jiang, X.W., Ding, L., Guan, Z.H., Yuan, F.S.: Bifurcation and chaotic behavior of a discrete-time Ricardo-Malthus model. Nonlinear Dyn. 71(3), 437-446 (2013)

16. Jiang, X.W., Zhang, X.S.: Stability and Neimark-Sacker bifurcation analysis for a discrete single genetic negative feedback autoregulatory system with delay. Nonlinear Dyn. 76(2), 1031-1039 (2014)

17. Kopel, M.: Simple and complex adjustment dynamics in Cournot duopoly model. Chaos Solitons Fractals 7(12), 2031-2048 (1996) 
18. Kopel, M.: Improving the performance of an economic system: controlling chaos. J. Evol. Econ. 7, 269-289 (1997)

19. Kuznetsov, Y.A.: Elements of Applied Bifurcation Theory, 3rd edn. Springer, New York (2004)

20. Li, B., He, Q.Z.: Bifurcation analysis of a two-dimensional discrete Hindmarsh-Rose type model. Adv. Differ. Equ. 2019, $124(2019)$

21. Li, B., He, Z.M.: Bifurcations and chaos in a two-dimensional discrete Hindmarsh-Rose model. Nonlinear Dyn. 76(1), 697-715 (2014)

22. Li, B., He, Z.M.: 1:2 and 1:4 resonances in a two-dimensional discrete Hindmarsh-Rose model. Nonlinear Dyn. 79(1), 705-720 (2015)

23. Ma, J.H., Yang, W.H., Lou, W.D.: Research on bifurcation and chaos in a dynamic mixed game system with oligopolies under carbon emission constraint. Int. J. Bifurc. Chaos 27(10), 1750158 (2017)

24. Matouk, A.E., Elsadany, A.A., Xin, B.G.: Neimark-Sacker bifurcation analysis and complex nonlinear dynamics in a heterogeneous quadropoly game with an isoelastic demand function. Nonlinear Dyn. 89, 2533-2552 (2017)

25. Panchuk, A., Puu, T.: Oligopoly model with recurrent renewal of capital revisited. Math. Comput. Simul. 108, 119-128 (2015)

26. Pecora, N.: Analysis of 1:4 resonance in a monopoly model with memory. Chaos Solitons Fractals 110, 95-104 (2018)

27. Puu, T.: Oligopoly: Old Ends-New Means. Springer, Berlin (2011)

28. Puu, T.: Disequilibrium Economics. Springer, Cham (2018)

29. Ren, J.L., Yu, L.P.: Codimension-two bifurcation, chaos and control in a discrete-time information diffusion model. J. Nonlinear Sci. 26(6), 1895-1931 (2016)

30. Rionero, S., Torcicollo, I.: Stability of a continuous reaction-diffusion Cournot-Kopel duopoly game model. Acta Appl. Math. 132, 505-513 (2014)

31. Torcicollo, I.: On the dynamics of a non-linear duopoly game model. Int. J. Non-Linear Mech. 57, 31-38 (2013)

32. Tremblay, C.H., Tremblay, V.J.: Oligopoly games and the Cournot-Bertrand model: a survey. J. Econ. Surv. 33(5), 1555-1577 (2019)

33. Vandermeer, J.: Period 'bubbling' in simple ecological models: pattern and chaos formation in a quartic model. Ecol. Model. 95(2-3), 311-317 (1997)

34. Wiggins, S.: Introduction to Applied Nonlinear Dynamical Systems and Chaos. Springer, New York (2003)

35. Wu, W.J., Chen, Z.Q., Ip, W.H.: Complex nonlinear dynamics and controlling chaos in a Cournot duopoly economic model. Nonlinear Anal., Real World Appl. 11, 4363-4377 (2010)

36. Yu, W.S., Yu, Y.: The stability of Bayesian Nash equilibrium of dynamic Cournot duopoly model with asymmetric information. Commun. Nonlinear Sci. Numer. Simul. 63, 101-116 (2018)

37. Yue, D.D., Guan, Z.H., Chen, J., Ling, G., Wu, Y.H.: Bifurcations and chaos of a discrete-time in genetic regulatory networks. Nonlinear Dyn. 87, 567-586 (2017)

38. Yuri, A.K., Hil, G.E.M.: Numerical Bifurcation Analysis of Maps. Cambridge University Press, Cambridge (2019)

39. Zhang, Y.F., Gao, X.: Equilibrium selection of a homogeneous duopoly with extrapolative foresight. Commun. Nonlinear Sci. Numer. Simul. 67, 366-374 (2019)

\section{Submit your manuscript to a SpringerOpen ${ }^{\circ}$ journal and benefit from:}

- Convenient online submission

- Rigorous peer review

- Open access: articles freely available online

- High visibility within the field

- Retaining the copyright to your article

Submit your next manuscript at $>$ springeropen.com 